\title{
"PARA ALGO QUE ERA NUESTRO... AHORA ES DE TODA LA HUMANIDAD”: EL PATRIMONIO MUNDIAL COMO EXPRESIÓN DE CONFLICTOS
}

\author{
FOR SOMETHING THAT WAS OURS... NOW IT BELONGS TO ALL OF \\ HUMANITY”: WORLD HERITAGE AS THE EXPRESSION OF CONFLICTS
}

\author{
Beatriz Santamarina1 y Camila Del Mármol
}

\begin{abstract}
En este artículo nos interesa reflexionar sobre la naturaleza conflictiva del patrimonio, que suele ser silenciada por su potente imaginario como capital cultural, económico y social y como signo de distinción. A través de un análisis detallado del proceso mediante el cual el tango y las fallas pasaron a formar parte de la Lista Representativa del Patrimonio de la Humanidad, nos adentramos en las dimensiones hegemónicas y de los procesos patrimoniales, así como en la esencia beligerante de las resistencias locales que se enfrentan a las lógicas del patrimonio neoliberal. Estos casos nos permiten vislumbrar las relaciones conflictivas que a menudo se establecen entre distintas lógicas, intereses y agentes de la patrimonialización, y nos permiten trazar puentes que contextualizan estos procesos en marcos políticos y de mercado más amplios. Más allá de las distancias y diferencias, podemos observar cómo el impulso de ambas candidaturas está en relación directa con las políticas de redefinición urbana, conocidas como New Urban Policy (NUP). La mundialización neoliberal articulada en la economía de los intangibles y en la industria cultural y del ocio y concretada en la ciudad creativa dibuja nuevos espacios urbanos sobre viejos lugares, ocupando el patrimonio inmaterial un lugar cada vez más destacable.
\end{abstract}

Palabras claves: Patrimonio Mundial, Patrimonio Inmaterial, tango, fallas, patrimonialización.

In this article, we discuss the conflicting nature of heritage which is usually silenced by its powerful representation as a cultural, social and economic capital, and as a sign of distinction. We will focus on tango and fallas and how they both became registered in the Representative List of Intangible Heritage. Based on those cases, we explore the hegemonic dimension of heritage processes as well as the bellicose local resistances that confront the logic of neoliberal heritage. The examples throw some light on the conflicts that may arise between the different interests, logics and agents involved in heritage processes. They also reveal the nature of the political and economic contexts that make the heritagisation possible. Beyond the distances and specificities of our two cases, they expose how the two nominations coincide with specific policies of urban regeneration, known as the New Urban Policy (NUP). Neoliberal globalization articulated around the intangible economy and the cultural and leisure industry take shape within the smart cities which redefine old places into new urban spaces. Intangible heritage plays a key role in these processes.

Key words: World Heritage, Intangible Heritage, tango, fallas, heritage processes.

En 2003 la Unesco viraba en sus políticas patrimoniales dando entrada al patrimonio inmaterial en su seno (Convención para la Salvaguardia del Patrimonio Cultural Inmaterial de París). Desde el 2006 la Lista Representativa del Patrimonio Cultural Inmaterial de la Humanidad se ponía en marcha con la incorporación de los bienes declarados hasta ese momento como Obras Maestras del Patrimonio Oral e Intangible (2001, 2003 y 2005). En poco más de una década el crecimiento de las declaratorias ha sido exponencial, coincidiendo con la hiperinflación o burbuja patrimonial (Bendix 2009; Heinich 2009). En muchos lugares han celebrado su incorporación a la lista de Patrimonio Inmaterial de la Humanidad, programando actos festivos y demostraciones. En Buenos Aires se celebró la inscripción del tango con una Gran Milonga Abierta en la emblemática esquina de San Juan y Boedo ${ }^{1}$. En el caso de Valencia, en 2016

\footnotetext{
${ }^{1}$ Universidad de Valencia, Valencia, España. Beatriz.Santamarina@uv.es

${ }^{2}$ Universidad de Barcelona, Barcelona, España. cdelmarmol@ub.edu

Recibido: julio 2018. Aceptado: julio 2019.
}

http://dx.doi.org/10.4067/S0717-73562020005000301. Publicado en línea: 16-enero-2020. 
celebraron la incorporación de las Fallas a la lista con la plantá de una falla en la Torre de Serranos, lugar especialmente simbólico ${ }^{2}$, que fue quemada en una gran fiesta posterior ${ }^{3}$.

La transformación de la racionalidad de la economía neoliberal ha traído consigo la creciente mercantilización de la cultura y el ocio, la expansión de la industria del turismo global y el despliegue de la gestión de los intangibles (Anheier y Isar 2008; MacCannell 1999; Scott 2007; Throsby 2001). En este contexto de mundialización neoliberal, el desembarco del patrimonio inmaterial ha sido aplaudido porque responde a la perfección a la lógica y las demandas de un mercado globalizado ansioso por consumir autenticidad (Boltanski y Chiapello 2002; Comaroff y Comaroff 2009; Davallon 2010; Frigolé 2014; Harvey 1998; Santamarina y Moncusí 2015). De hecho, hoy podemos afirmar que el mercado dispone de lo patrimonial como un activo económico de primer orden (Ashworth y Van Der Aa 2006; Brumann 2012; Meskell 2013, 2014; Schmitt 2008). Más allá de los explícitos intentos de la Unesco para que sus acciones incidan positivamente en la conservación, salvaguardia y restauración de los bienes designados como patrimonio, lo cierto es que cada vez más las declaratorias han promovido la producción de nuevos valores mercantiles. De esta forma, la organización ha jugado un papel central como productora y certificadora de un sello de calidad patrimonial global. Esta institución, como ha señalado Meskell (2014), más que una agencia para la conservación o salvaguardia patrimonial es hoy un organismo para otorgar marca.

Si bien los medios de comunicación de distintos países y continentes han reflejado el júbilo por conseguir tan alta distinción -basta con asomarse a los distintos titulares en la prensa escrita- no es menos cierto que las declaratorias han puesto encima de la mesa las grietas y fisuras de la concepción y expansión del Patrimonio Mundial. Más allá de las críticas efectuadas desde la academia y desde el propio seno de la Unesco (Askew 2010; Brumann 2012; Frey et al. 2013; Labadi 2007; Meskell 2013; Meskell y Brumann 2015; Pavone 2008; Schmitt 2008), otras voces han cuestionado las declaratorias mundiales. Voces locales, experiencias arraigadas que nos acercan a otras derivas de las expresiones culturales, antes y más allá de la patrimonialización.

En 2009, Argentina y Uruguay vivían con intensidad la incorporación del tango a la Lista de Patrimonio Inmaterial. De todos los comentarios y declaraciones que resultaron de esta proclamación, quizás el que nos parece más revelador de la naturaleza de los temas implicados fue el reproche velado de un tanguero argentino que, en un concierto realizado en el Café Vinilo ${ }^{4}$ en 2009, espetó a sus oyentes: "Carajo, para algo que era nuestro, ahora es de toda la humanidad". La crítica, compartida con muchos músicos del tango, venía a decir que el tango es porteño, no de la humanidad. En realidad, el reproche se hacía eco de la posición del Sindicato Argentino de Músicos que, bajo el lema "El tango ya no es nuestro", se posicionó en contra de la candidatura (Sindicato Argentino de Músicos 2009) Por tanto, no se trataba de un comentario inocente, el enunciado remitía a la expropiación y reapropiación en términos patrimoniales en consonancia con la postura del sindicato de músicos. El conflicto afloraba como una queja, con gusto a tango, que contenía el problema latente de la edificación patrimonial. Ya desde principios de siglo, asambleas y agrupaciones diversas se organizaron para oponerse a las tendencias de la política cultural de la Ciudad de Buenos Aires, que se concretaban en proyectos de privatización como la Ley de Mecenazgo y otras iniciativas que se orientaban, según sus críticos, a convertir al tango en un producto para atraer el turismo (Morel 2013). "Autoconvocados por el Tango" se organiza en 2001 como reacción a las políticas del gobierno de la Ciudad que promueven una transformación en clave mercantil del "Festival Buenos Aires Tango", denunciando su mercantilización y su utilización como un activo turístico (Morel 2009, 2013). El movimiento \#ElTangoNoSeClausura, el colectivo cultural "Quinteto Negro La Boca", o el proyecto del "Festival de Tango Independiente", son otras de las plataformas, asambleas, asociaciones e iniciativas que a lo largo de los años y hasta la actualidad se organizan para oponerse a diferentes procesos que apuntan a la mercantilización del tango, a la privatización y espectacularización de sus manifestaciones, así como a las clausuras de milongas por parte del Gobierno de la Ciudad.

En un continente distinto, y unos años después, se repetía la misma explosión de alegría en España (Comunidad Valenciana) con la declaración del Espacio Cultural de las Fallas (2016) como Patrimonio Mundial. Mientras las autoridades políticas y el mundo festero celebraran la codiciada distinción, el Colectivo Falles Populars i Combatives creaba el club Patrimoney y daba vida a un particular personaje sarcástico, 'La Patri', en enero del $2016^{6}$, gracias a una campaña de crowdfunding $^{7}$. Esta figura imaginativa tenía una misión particular: proteger allí por donde pasaba. En realidad, La Patri, diminutivo del nombre \#LAPATRIMONIALITZADORA, era un particular monumento fallero. El mismo era móvil, nómada según sus creadores, gracias a una plataforma con ruedas. $\mathrm{La}$ Cooperativa Crearqció, encargada del proyecto, realizó la obra a la que definió, significativamente, como un 'Monumento Itinerante de Activación Urbana'. En el proyecto, se señalaba que el objetivo era crear "Una falla que anda y que no le hace falta que nadie vaya a verla. 
Ella es independiente para ir a ver y saludar a todo el mundo" . De tal manera que el ingenio y la gracia (ingeni i gràcia en valenciano), propios de los monumentos falleros, junto con la sátira que caracteriza estas fiestas, ponían el dedo en la llaga sobre la mercantilización de la fiesta y advertían que no necesitaban el respaldo de la Unesco para vivir su fiesta. La crítica compartía con los tangueros su reivindicación de que las fallas eran fiestas locales, no de la humanidad, y el riesgo de someter la cultura a las reglas del mercado. La desaprobación de la distinción, con lenguaje fallero, contenía los peligros de la inflación de la fábrica patrimonial. Este colectivo había empezado su andadura en el $2002^{9}$, coincidiendo con la apuesta de las políticas públicas valencianas de iniciar numerosos programas de intervención en la ciudad, que empezaron a transformar su imagen y su fisonomía rápida y substancialmente (Boix et al. 2017).

La frase de nuestro tanguero y el personaje de nuestros alternativos falleros iluminan toda una serie de resistencias patrimoniales. En apenas una decena de palabras, nuestro músico, reconvertido en espontáneo crítico patrimonial, sacaba a luz la esencia beligerante de las distintas formas patrimoniales. La nostalgia sobre el pasado y sobre la pertenencia, "era nuestro" (de nos-otros), eran explícitos, un nuestro además que se subraya como vertebrador de identidad (lo propio, lo característico). Por otra parte, La Patri, esa figura ingeniosa, nacida de la sátira y la agudeza característica de las fiestas falleras, cobraba vida como elemento desestabilizador. La censura sobre las formas hegemónicas de mercadeo patrimonial, encapsuladas en el Patrimoney, advertían sobre la deriva del patrimonio neoliberal. Y ponían sobre la mesa la relación conflictiva entre los diferentes intereses, lógicas y agentes patrimoniales. Entendemos aquí por patrimonio neoliberal aquel que se activa fundamentalmente para insertarse y posicionarse en las redes de los mercados transnacionales y está sujeto a los dictámenes e intereses del tercer espíritu del capitalismo (Boltanski y Chiapello 2002).

En este artículo nos interesa reflexionar sobre la naturaleza conflictiva del Patrimonio Mundial, que suele ser silenciada por su potente imaginario como capital cultural, económico y social y como signo de distinción(Bourdieu 1988, 1991; García Canclini 1993). En pleno auge del patrimonio colectivo, su discurso es utilizado en diferentes ámbitos para legitimar distintos reclamos y revestir diversas luchas de poder. Los conflictos en el ámbito patrimonial son recurrentes en la medida en que entran en juego lógicas dispares y en competencia. En este sentido, el aparato patrimonial puede ser visto como lugar de conflicto, en la medida que participa en la definición de las realidades sociales y políticas. Pero también sirve como un campo donde las disputas sociales se expresan y dónde es posible observar las mismas fracturas, tensiones, posiciones y diferencias internas inherentes a cualquier comunidad local y en todas las escalas de actores. A partir de dos casos de estudio, el tango y las fallas, queremos poner el acento en dos de los problemas interrelacionados del Patrimonio Mundial: la propiedad y el mercado. Ambos obligan a abrir una reflexión sobre el peligro de la fetichización, espectacularización y mercantilización del patrimonio. El estudio del tango se basa en un trabajo de campo etnográfico realizado en Buenos Aires en 2014, complementado con algunas visitas que se extienden hasta 2017. El objetivo era hacer una prospección del desarrollo de políticas culturales porteñas en materia de patrimonio cultural inmaterial, especialmente aquellos proyectos referidos a la inscripción de elementos de patrimonio inmaterial en las listas de la Unesco. En el caso de las fallas, este trabajo se inserta en dos investigaciones más amplias, una sobre las transformaciones de la ciudad de Valencia, atendiendo a sus distintas activaciones patrimoniales, y otra sobre movimientos urbanos en una barriada en concreto de dicha capital, desarrolladas de forma continuada entre 2009 y 2017. En los dos casos se han utilizado distintas herramientas metodológicas: conversación (entrevistas a informantes clave), observación (escenarios privilegiados) y análisis de contenido (prensa, legislación y producciones activistas). La estrategia del análisis para este trabajo responde a la teoría fundamentada (Grounded Theory) para identificar procesos sociales básicos a través del análisis de información (codificación y categorización) y el método comparativo (Carrero et al. 2006).

\section{El Imperialismo Patrimonial: de la Identidad al Mercado}

La Convención para la Protección del Patrimonio Mundial, Cultural y Natural (Unesco1972) transformaba la lógica nacional que regía al patrimonio hasta el momento. La versión clásica del "nosotros del nosotros" patrimonial no se rompía, sino que daba un paso firme hacia adelante transformándose definitivamente en un "nosotros para los otros"(Prats 1997). Una versión identitaria con proyección exterior, que tras la Segunda Guerra Mundial había ido configurándose con la llegada del ocio, la democratización del turismo y la revolución de los transportes. Más que un patrimonio mundial se establecía un patrimonio para consumo mundial, como pronto se puso de manifiesto. Si el liberalismo, el nacionalismo y el capitalismo habían dado lugar al patrimonio decimonónico, las nuevas condiciones de producción con el neoliberalismo, la globalización y el neocapitalismo posibilitaron el Patrimonio Mundial. Así, es posible afirmar que, si bien el patrimonio como uso del pasado en el siglo XIX se relaciona con los 
procesos de producción de la nación, hoy podemos decir que se inserta en redes transnacionales vinculadas a los mercados (Frigolé 2014; Roigé y Frigolé 2010; Santamarina y Del Mármol 2017). Este cambio es fundamental para entender el viraje emprendido por el patrimonio colectivo: de las topografías nacionales hemos basculado hacía las topografías turísticas. Y en ese movimiento la Unesco ha ejercido un papel sobresaliente creando la marca de Patrimonio Mundial.

El sello Unesco, como firma distintiva del producto patrimonial, adquirió pronto un valor extraordinario desplazando su original concepción como mecanismo de conservación. De hecho, la marca desplazaba al patrimonio, en al menos dos sentidos. Por un lado, ya no era el bien lo que debía ser único para su activación, siguiendo la lógica de la escasez (Cruces 1998), sino que también la marca debía lograr ser única como sello que imprimiera la diferencia de la competencia. Por otro, el binomio patrimonio/identidad era sustituido por marca/ identidad, lo importante era ofrecer una lectura única y significativa de la imagen añadiendo una estimulación hacia su consumo. La Unesco dotaba de significados a su marca, enriqueciendo el producto patrimonial y ofreciendo al consumidor pasados e identidades listas para ser consumidas. El marketing patrimonial y las estrategias mercadológicas se ponían al servicio de la promoción de los polos turísticos.

La potente marca de Patrimonio Mundial instaurada en 1972, junto a la incorporación del Patrimonio de la Humanidad en 2003, siguen hoy rigiendo las lógicas globales patrimoniales, vinculadas a las nuevas demandas del tercer capitalismo (Boltanski y Chiapello 2002) y la expansión del sector turístico. Entrar en las listas de la Unesco supone tanto un sello de calidad, legible y compartido globalmente, como una distinción que permite competir por los circuitos mercantiles y los mapas de la excelencia turística. Hasta el punto que algunos autores señalan a la "Unesco as an agency for global branding rather than global conservation" (Meskell 2014:217). Pero lo que nos interesa subrayar, es que las listas de la Unesco entrañan dos dimensiones sobre la propiedad: una jurídica y otra simbólica (Benhamou 2010) que abre los procesos globales patrimoniales a conflictos, resistencias y acomodaciones de diferente calado. En cuanto a la dimensión jurídica, la noción de bien público global implica cumplir una serie de derechos y obligaciones marcados por la aceptación de los marcos normativos de las convenciones ${ }^{10}$. Por lo que respecta a la simbólica, la propiedad del bien vinculada a un territorio local y articulada en narrativas situadas salta a un espacio desterritorializado donde la modulación se establece por ficciones desplazadas.

Entrar en la lista de Patrimonio de la Humanidad supone un ejercicio complejo y contradictorio, al menos, en términos discursivos. La identidad, el territorio y la memoria necesitan redefinirse para adaptarse a los sentidos del aparato patrimonial global. El salto es considerable y las tensiones no tardan en aparecer. Todo esto no es nuevo. Desde su constitución, y por su propio carácter sociopolítico, lo patrimonial ha resultado siempre conflictivo. En su versión decimonónica, la construcción de la nación necesitaba de la producción de un patrimonio colectivo. La creación de una comunidad imaginada (Anderson 1983), requería el establecimiento de una memoria compartida encapsulada en la tradición. El pasado institucionalizado, en su armadura patrimonial, implicaba la selección y jerarquización de una narrativa única, un lugar delimitado con el fin de instrumentalizar una versión identitaria propia. Su condición intrínseca de disputa, por su propiedad maleable, convertía al pasado recién inaugurado en una arena susceptible de ser utilizada por distintos actores dando lugar a interpretaciones divergentes y abierto con ello a la pugna.

En este texto nos situamos en la perspectiva crítica del patrimonio que abrió el análisis a su carácter conflictivo (Bendix et al. 2012; Davallon, 2010; Del Mármol 2012; Harrison 2013; MacDonald 2013; Poulot 2006; Smith 2006). En el mismo, el conflicto se presenta en términos políticos y de confrontación entre diversos agentes por la apropiación legitima del campo patrimonial. Siguiendo a Smith (2006), las distinciones hegemónicas se transforman en discursos patrimoniales autorizados, por lo que podemos tratar los sentidos preceptivos y performativos como campos donde se escenifican tanto las distintas estrategias y agentes como la estructura de la distribución asimétrica de sus capitales (Bourdieu, 1991, 1994) ${ }^{11}$.

El patrimonio colectivo siempre ha sido un campo de conflicto y negociación porque a través de él se ha dado sentido al pasado configurando el presente (Herzfeld 1991; Lowenthal 1998). En la producción de usos del pasado se escenifican magistralmente las relaciones de poder, de ahí su carácter político como dispositivo de definición de realidades. Su eficacia simbólica ha residido en la capacidad de presentar los usos del pasado como realidades naturalizadas, un sistema de hechos que se derivan sin más del recuerdo de aquello que fue. Esta facultad de presentarse como sustantivo, alejado de sus propias condiciones sociales de producción, y reconfortado en sus propiedades estéticas también esencializadas, le confiere una extraordinaria potencialidad como instrumento político. Además, su temprana canalización hacia las vertientes técnicas y disciplinares (conservación, restauración, etc.) desvió la atención de su eminente dimensión política, su carácter gubernamental (Santamarina y Beltran 2016).

En la actualidad, el patrimonio ha sido largamente criticado como un aparato hegemónico de dominación y control, en un sistema mundo-colonial y en el contexto de una economía mundial específica (Smith 
2006; Bendix et al. 2012). Pero también ha sido entendido como un instrumento de oposición en términos de su potencial contra-hegemónico (Collins 2008; De Cesari 2010). Hemos apuntado algunas consideraciones referidas a lo primero, aunque habría que hilar más fino para entender el complejo de la globalización patrimonial, como, por ejemplo, el sistema de participación y la toma de decisiones de la Unesco que refleja fielmente las estructuras de poder de otros organismos mundiales (Frey et al. 2013; Meskell et al. 2015). En todo caso, el patrimonio debe entenderse siempre en términos procesuales, como un conjunto de discursos y prácticas que transforman las condiciones sociales de producción de determinadas realidades sociales. Y es, en este sentido, en el que se abre como un campo proclive al conflicto. Cuando el tanguero expresa su lamentación abre la vía para oponerse a las políticas no sólo globales sino también nacionales y locales. Lo mismo sucede en el caso valenciano, cuando un grupo, el colectivo Falles Populars i Combatives, inicia una campaña en contra de la declaración de la fiesta como Patrimonio de la Humanidad. Veamos entonces cómo se han articulado ambas prácticas y cómo se han modulado estas resistencias.

\section{El Tango y las Fallas: de las Redes Vecinales a las Políticas Globales}

De forma sintética, y antes de comenzar nuestro análisis, describiremos brevemente los bienes a los que nos referimos. El tango comprende un estilo de música, una danza y una poética y es propio de la cuenca del Río de la Plata. A finales del XIX, se desarrolló, principalmente, en los suburbios de las ciudades de Buenos Aires y Rosario (Argentina) y Montevideo (Uruguay). Su origen es expresión de una hibridación cultural entre diferentes ritmos musicales de emigrantes europeos, descendientes de esclavos africanos y criollos. La expresión del tango comprende a una multitud de actores desde músicos, hasta bailarines pasando por coreógrafos, compositores y letristas ${ }^{12}$. La historia del tango a lo largo del siglo XX atraviesa diferentes etapas de expansiones y declives, hasta llegar a un aumento progresivo de su popularidad a partir de los años $90 \mathrm{y}$ hasta la actualidad. En este contexto, se revitalizan las milongas barriales, espacios de baile y sociabilidad que vivieron un primer auge en la década de 1940, así como las orquestas y músicos del género.

Por su parte, las fallas son una fiesta de celebración de la llegada de la primavera que se practica, como tal, desde finales del XIX. Cada año entre el 15 y el 19 de marzo se instalan en las calles y plazas de Valencia grupos escultóricos artesanales (falles), compuestos por muñecos (ninots) de cartón piedra y sostenidos por una estructura de madera. Los artistas falleros trabajan todo el año en la construcción de las fallas. Estos monumentos, con carácter satírico sobre temas de actualidad, se queman (cremà) el último día, en honor a San José, patrón de las fiestas. Las fallas ${ }^{13}$ son plantadas por las distintas comisiones falleras que se reúnen a lo largo del año en locales (casals falleros), distribuidos por todos los barrios de la ciudad, organizando actos festivos y culturales que, más allá de buscar recursos para la financiación de las fiestas, funcionan como lugares de sociabilidad de primer orden en la ciudad. Además, estas fiestas se caracterizan por el uso de la pólvora y pirotecnia y la música tradicional y de bandas que acompañan todas las jornadas festivas ${ }^{14}$.

La elección del tango porteño (Argentina) y las fallas valencianas (España) para nuestra reflexión no ha sido aleatoria. En dos continentes distintos (América y Europa), con candidaturas diferentes, una biestatal (Argentina y Uruguay) ${ }^{15}$ y otra estatal (España), con recorridos diversos de éxito y fracaso (el tango no fue reconocido como Obra Maestra en el primer intento de ser inscrito) y representando a bienes de distinta tipología (artes de espectáculo y actos festivos). Pero, además, ambas designaciones responden a contextos patrimoniales mundiales inversos y a mercados turísticos alejados. Por lo que respecta al patrimonio podemos decir que Argentina está subpatrimonializada, tiene en total 13 bienes mundiales, sumando las dos listas Unesco (seis bienes culturales, cinco naturales y dos inmateriales). En cuanto a la industria turística ocupa la posición 50 dentro del ranking de países que reciben turistas según la Organización Mundial del Turismo (UNWTO 2017). Por su parte, España pese a ser un territorio mucho menor en extensión $\left(504.645 \mathrm{~km}^{2}\right.$ frente a $3.761 .274 \mathrm{~km}^{2}$ de Argentina), está sobrepatrimonializada, ostentando 62 bienes mundiales entre las dos listas ( 40 bienes culturales, cuatro naturales, dos mixtos y 16 inmateriales) y ocupando el tercer puesto en número de declaratorias de Patrimonio Mundial tras Italia y China. Asimismo, según la OMT, ocupó el tercer puesto como destino turístico en 2016 y el segundo puesto en cuanto ingresos turísticos (UNWTO 2017:6).

Pero, pese a las diferencias notables entre un país y otro, podemos decir que ambos comparten el mismo lenguaje patrimonial y, en los dos casos, ha habido políticas patrimoniales que han instrumentalizado las prácticas del tango y las fallas para fines gubernamentales y de mercado. Según los intereses de cada momento, esas intervenciones las han normalizado, cosificado y dirigido, hasta convertirlos en los bienes patrimoniales de primer orden nacionales e internacionales.

En el caso argentino, en las primeras décadas del siglo XX, el tango empieza a identificarse con lo porteño, pasando de los arrabales y las orillas proscritas, espacios malignos y peligrosos, a la ciudad y convirtiéndose en "un actor importante en la batalla por la producción de una subjetividad significante" (Vila 2000:71). 
Pese a las primeras resistencias de la oligarquía, el tango, tras un proceso de depuración y limpieza, será institucionalizado neutralizando su contenido subversivo y "convirtiéndolo en un fenómeno estético" (Matamoro 1982:73). De esta forma, pronto será asumido por las élites e instrumentalizado para la reconstrucción política y moral de la ciudad y de la nación. Aparecen, en este periodo, los tangos de reafirmación de la patria y de los símbolos nacionales, de la historia y la identidad argentina (Varela 2005; Vila 2000). Tal y como señala Vila (2000) el tango, como artefacto cultural complejo, es utilizado para construir una idea de la argentinidad al ofrecer la superposición de distintos códigos para la construcción de identidad poniéndose al servicio del interés hegemónico del momento. En esta época, Buenos Aires es configurada como la ciudad del tango y el tango como la música de la ciudad comercial (Matamoro $1982)^{16}$. Durante las décadas de los 40 y 50 , en la llamada etapa de dorada del tango (Pelinski 2000), cuando se hace masivo y popular, la geografía del tango se ha transformado y se ha instalado "en la vida de los barrios" (Cecconi 2009:58) contribuyendo a la construcción de la identidad porteña (Vila 2000) de una ciudad ya industrial (Matamoro 1982). Tras un periodo, las décadas del 60 y 70 , calificado por algunos como de crisis del tango por su desaceleración social y su transformación vanguardista, a final del siglo pasado, sobre todo, a partir de los ochenta se empezarán a implementar políticas de recuperación del tango (Gómez et al. 2011; Morel 2012). Aunque será a finales de 1990 cuando aparezcan las primeras normas en torno al tango. Primero fue el Estado argentino quién con la Ley Nacional 24.684, conocida como la Ley del Tango (Boletín Oficial, 2 de septiembre de 1996) ${ }^{17}$ declaraba en 1996 el tango como parte integrante del patrimonio cultural de la Nación. Dos años más tarde fue la Ciudad Autónoma de Buenos Aires quien puso en marcha su propia normativa con la Ley $n^{\circ} 130$ sancionada en 1998 (Decreto 37/99 del 14 de enero de 1999, Boletín Oficial de Buenos Aires n $\left.{ }^{\circ} 616\right)^{18}$. Ambas regulaciones se encuentran enmarcadas en un contexto más amplio de iniciativas legislativas que comienzan a finales del siglo XX y principios del XXI en torno a la promoción y protección del tango (Morel 2009).

En el caso español, el esquema se repite, con una intervención nacional y local por la fiesta, no así el procedimiento por los propios contextos diferenciables entre ambos países. A finales del siglo XIX, las fallas comienzan a ser una fiesta arraigada en la ciudad y serán pronto neutralizadas por la burguesía local. Los aspectos más formales de la fiesta se van definiendo y los monumentos de marcado carácter de crítica política van transitando hacia los monumentos artísticos. Surgen así las fallas que exaltan las glorias de la patria local y española que irán transformándose, en las primeras décadas del XX, en un elemento central de la identidad valenciana (Ariño 1992a, 1992b; Hernàndez 2006). Las fallas, como liturgia civil del valencianismo (Ariño 1992b), fueron instituidas como un instrumento de afirmación del sentimiento identitario de lo valenciano al servicio del Estado español ${ }^{19}$. Durante la dictadura franquista (1939-1975), el Estado fomentó la fiesta fallera por sus lazos con el ruralismo tradicional y como propaganda del régimen (Hernàndez 1996; Obiol 2009). En este sentido, Hernàndez (1996) analiza cómo el franquismo utilizó las fallas para cimentar sus políticas nacionales. En 1946 las fallas fueron declaradas Fiestas de Arte de interés nacional (Orden del 14 de marzo de 1946, Boletín Oficial del Estado $\left.\mathrm{n}^{\mathrm{0}} 75\right)^{20}$, reconociéndose su carácter artístico y su asentamiento en la ciudad, así como su atracción como polo turístico. En los años 1960, cuando el turismo se convirtió en una política prioritaria en España para salir de la autarquía, se creó una distinción honorífica bajo la denominación de Fiesta de Interés Turístico ${ }^{21}$ con el fin de promoverlo. El Estado declaró las fallas como Fiesta de Interés Turístico en 1965 (Boletín Oficial del Estado $\left.\mathrm{n}^{\mathrm{o}} 35\right)^{22}$. Más tarde, ya en democracia y con una nueva tipología de distinciones, fueron reconocidas como Fiesta de Interés Turístico Internacional en 1980 (Boletín Oficial del Estado $\left.{ }^{\circ} 41\right)^{23}$. Tras estos impulsos estatales a las fiestas, convertidas ya en un referente nacional, la nueva patrimonialización sobre los bienes inmateriales a raíz del Programa de Obras Maestras del Patrimonio Oral e Intangible de la Humanidad (1998) permitió la activación autonómica de la figura de Bien de Interés Cultural Inmaterial (BICI). El gobierno local declaró a las fallas como BICI en el 2012 (Decreto 44/2012, de 9 de marzo del Consell, Diario Oficial Comunidad Valenciana $n^{\circ}$ $6732)^{24}$ como paso previo a su reconocimiento mundial.

De cualquier manera, pese a que las trayectorias de reconocimiento y de intereses de ambos bienes hayan sido diferentes, en los dos casos, ha habido una instrumentalización nacional necesaria para su distinción como Patrimonio de la Humanidad. Tal y como señalan Ashworth y Van der Aa (2006) la lista de patrimonio refleja mucho más los intereses nacionales que los globales, no hemos de olvidar que la Unesco es una organización de naciones ${ }^{25}$. Pero, además, el tango y las fallas comparten tres rasgos fundamentales. En primer lugar, en ambos casos se produce una apropiación hegemónica que reconduce sus aspectos más subversivos hasta transformarlos en operadores de orden institucional. En el tango, algunos autores han llegado a calificar este proceso de gentrificación ${ }^{26}$ (Cecconi 2009), por su asunción por la clase dominante que domesticó sus aspectos más desestabilizadores asociados a la marginalización y el desorden. En las fallas el control de los elementos más peligrosos, por el carácter transgresor y crítico de sus monumentos, por parte de la burguesía ilustrada, fue clave para su consolidación e institucionalización (Ariño 1992a; Hernàndez 2002, 2006). En ambos casos, de los sectores 
más populares impulsores de estas prácticas se pasa a la apropiación de las élites adaptándolos a sus gustos y al servicio de la ideología imperante. De los prostíbulos y los arrabales de los márgenes porteños a los teatros y cabarets del centro de Buenos Aires operan toda una serie de mecanismos de usurpación que reconfiguran el tango (Matamoro 1982; Pelinski 2000; Varela 2005; Vila 2000). Del mismo modo, de las fiestas reprimidas e impulsadas por las clases populares, marginadas éstas en el sistema sociopolítico, a la formalización organizativa de la fiesta y la llegada de los premios institucionales suceden toda una cadena de confiscaciones que redefinen las fallas (Ariño 1992a; Hernàndez 2002, 2006). El carácter subversivo del tango y las fallas queda contenido a través del control disciplinario del aparato político y en ambas prácticas su dulcificación pasa por el refinamiento de la estética del baile y los monumentos, respectivamente.

En segundo lugar, ambos bienes han funcionado como importantes catalizadores de identidad, de ahí las disputas por sus sentidos y su utilización como arma política. Tanto en el tango como en las fallas se han producido intentos de monopolización en distintas etapas políticas y por diferentes partidos políticos que han utilizado sus expresiones con fines partidistas. Igualmente, en los períodos de dictadura de Argentina y España ha habido un control férreo sobre las mismas, activándose mecanismos censores y autoritarios en ambos casos (Ariño 1992a; Hernàndez 2006, 2011; Matamoro 1982; Varela 2005). Y, en tercer lugar, es necesario tener presente que ambos bienes son expresiones producidas, fundamentalmente, en contextos urbanos. Si el tango se instala en la vida de los barrios (Cecconi 2009; Morel 2009), lo mismo sucede en las fallas que son una fiesta de vecindad (Cucó 2008). Hoy, por hacernos una idea, hay más de cien milongas donde a diario se baila tango en Buenos Aires (Morel 2016) y trescientas noventa y una comisiones falleras repartidas por la ciudad de Valencia (Censo Junta Central Fallera) ${ }^{27}$. Esta particularidad es especialmente importante y ayuda a entender la propia diacronía de los bienes, así como la propuesta y el alcance de las candidaturas como Patrimonio de la Humanidad. Si a finales del XIX y principios del XX, el Buenos Aires portuario y la Valencia agrícola experimentan una remodelación urbana que refleja la transición hacia el mundo moderno y el ascenso de nuevas clases sociales y políticas; a finales del siglo XX y principios del XXI, el nuevo contexto de la economía global ha forzado a las grandes urbes, como Buenos Aires, o a las ciudades intermedias, como Valencia, a redefinirse, para recuperar el potencial de atracción que habían ejercido en el pasado como centros de producción industrial, lo que ha provocado una trasformación de sus fisonomías, sus espacios y sus narrativas ${ }^{28}$. La desregularización, privatización y flexibilización del mercado laboral, la descentralización espacial y financiera, la desindustrialización de las grandes áreas urbanas, la revolución de las tecnologías y transportes y la transformación del ocio ha obligado a los centros urbanos a adaptarse al nuevo contexto global para no quedarse fuera de las exigencias del mercado posfordista creando nuevas ficciones y activos para entrar en un mercado cada vez más agresivo.

En este contexto, y desde nuestro punto de vista, el impulso de ambas candidaturas está en relación directa con las políticas de redefinición urbana, conocidas como New Urban Policy (NUP). Tanto Buenos Aires como Valencia inician en la década de 1990 del siglo XX una transformación urbana en consonancia con el nuevo modelo hegemónico de metrópoli ligado a una nueva versión del urbanismo empresarial (Harvey 2001, 2013) o al llamado capitalismo cognitivo-cultural (Scott 2008, 2014). Estas políticas se modulan en notables proyectos arquitectónicos, grandes eventos mediáticos, desarrollo inmobiliario, privatización de fondos públicos y marketing urbano (Dinardi 2017; Swyngedouw et al. 2002) en busca de la competitividad y la excelencia para entrar en los circuitos globales (Connolly 2013; García 2004; Gold y Gold 2008; Rodríguez y Vicario 2005; Swyngedouw et al. 2002). La mundialización neoliberal articulada en la economía de los intangibles y en la industria cultural y del ocio y concretada en la ciudad creativa dibuja nuevos espacios urbanos sobre viejos lugares (Anheier y Isar 2008; Harvey 1998; Throsby 2001). Ocupando el patrimonio, por su capacidad de satisfacer la demanda, un lugar cada vez más destacable.

Buenos Aires experimenta una transformación significativa en la década del 90, impulsando grandes proyectos de renovación urbana, cuyo máximo exponente puede verse en la transformación de Puerto Madero (Cuenya 2011; Cuenya y Corral 2011; Díaz Orueta et al. 2003; Dinardi 2017). Valencia, por su parte, adoptó la tendencia global de la NUP a finales de los 90 (Cucó 2013a, 2013b; Rausell 2010; Rius et al. 2016; Santamarina y Moncusí 2013). El nuevo modelo de ciudad creativa, denominado por algunos como modelo Calatrava (Boix et al. 2017), puede verse encapsulado en la Ciudad de las Artes y las Ciencias. Ambas ciudades comparten características en su transformación, entre otras, han emprendido grandes proyectos urbanos asociados a procesos de gentrificación; han transformado su waterfront; han impulsado políticas de creación de marca con agencias especializadas para su desarrollo, buscando crear una imagen potente de las ciudades; y han apostado por distintas políticas patrimoniales, impulsando entre ellas las candidaturas a Patrimonio Inmaterial de la Humanidad buscando captar el turismo internacional (Bertoncello y Troncoso 2014; Díaz Orueta 2010; 
Gómez et al. 2011; Rausell 2006). En esa competencia por los mercados y los consumidores, las redes vecinales y de sociabilidad tejidas en sentidos locales han saltado a las políticas globales. Eso sí con resistencias.

\section{Resistencias al Patrimonio Mundial: Del Baile y lo Festivo a lo Combativo}

En el tango y las fallas se han dado procesos de resistencia en lugares muy concretos: los barrios de la ciudad de Buenos Aires y Valencia. El modelo hegemónico del patrimonio nacional o mundial es contestado desde la calle, el lugar precisamente donde se tejen las milongas y los casales falleros (espacios por excelencia de sociabilidad). Lo que permite ver desde el lugar (Escobar 2010), en los territorios barriales, otras lógicas patrimoniales construidas en la vida diaria y local y lejos del vocabulario administrativo y tecnocrático de los gobiernos y la Unesco.

Como acabamos de ver, el patrimonio, como fenómeno social y cultural, es siempre producido en un contexto específico que se ve alterado con el tiempo. Del tango del nosotros, de las milongas barriales de la capital porteña, al tango para ustedes, el escenario global donde se articulan las performances de identidad marcadas a ritmo de seducción (tango show), ha habido una transformación de acentos, agentes e intereses (Morel 2013). Lo mismo sucede con las fallas de Valencia, de las fallas de vecinos, comunitarias, solidarias y altruistas a las fallas monumentales globales, guiadas por los nuevos valores neoliberales ha habido un cambio significativo de cadencias, actores y capitales (Hernàndez 2011). Pese a la idealización del pasado que contiene esta última aseveración, con ella no negamos las fracturas y tensiones del pasado fallero, tan solo buscamos subrayar la ruptura cronotópica y gramatical que se ha producido. En cualquier caso, el conflicto no se reduce a la patrimonialización, sino que se sirve de este nuevo escenario para encontrar maneras de expresar la multiplicidad de posturas.

En Buenos Aires en 2014, un funcionario de la Subsecretaría de Patrimonio de la Ciudad de Buenos Aires celebraba la nominación del tango ya que había traído a muchos extranjeros al festival internacional: "se generó algo maravilloso, el hecho de que se reconozca de afuera hizo ver a la gente que éramos los representantes del tango" (entrevista junio 2014). Que el tango es porteño (o rioplatense, una concesión que se pudo hacer en nombre de una concordia nominativa) no es algo que se necesite recordar a los habitantes de Buenos Aires, es más interesante esta necesidad de legitimarlo ante una audiencia global.

La patrimonialización del tango nos muestra una historia de institucionalizaciones, normativizaciones y posteriormente un legado de iniciativas populares autogestionadas y reivindicativas que llegan hasta la actualidad. En 1998, como acabamos de señalar, Buenos Aires reconoce el tango como parte de su patrimonio cultural con la Ley $n^{\circ} 130$. Con ella creaba la Fiesta Popular del Tango, que desde sus inicios se conoció como el Festival Buenos Aires Tango y cambió su fecha de celebración hasta adaptarse a los flujos de turismo del hemisferio norte (Morel2009, 2013).Larealización de este mega evento organizado por la ciudad de Buenos Aires, buscaba, según Morel (2013), posicionar la pertenencia e identificación del tango con la ciudad ante la creciente celebración de festivales privados en distintos lugares del mundo. Algunos autores han señalado cómo el Gobierno de la Ciudad se decanta hacia las expresiones espectacularizadas del tango encaminadas a promocionar el mercado turístico (Gómez et al. 2011; Morel 2013). Distintos colectivos se organizarán para disputar visiones alternativas, otras formas de hacer, vivir y pensar el tango. Las resistencias han sido impulsadas por colectivos que promueven como ideología la autogestión y la independencia buscando espacios alternativos para sus reivindicaciones. En ellos encontramos una variada heterogeneidad de origen y clase social. Además de estos colectivos, el propio Sindicato Argentino de Músicos formado por más de 25.000 músicos, con variedad en cuanto perfil sociológico, como ya vimos, manifestó su oposición: "El tango ya no es nuestro"29.

Otro caso es el del "Quinteto Negro La Boca", que en su manifiesto nos ofrece una perspectiva estético-política de su constitución en 2008 como colectivo cultural con un decidido arraigue barrial, que pretende rescatar las memorias alternativas del tango, sus raigambres libertarias y anarquistas reivindicando su capacidad de narrar otras historias y construir nuevas identidades ${ }^{30}$. De aquí nace también un proyecto que comienza en $2010^{31}$, el Festival de Tango Independiente, autogestionado por los propios artistas y que posteriormente funcionó como impulsor de distintos festivales barriales e independientes. Entre sus valores, los espacios y artistas que participan del Festival Independiente abogan por la valorización del tango como factor de identidad cultural y regional, centrándose en la necesidad de promover las iniciativas contemporáneas de las nuevas generaciones del tango y de implementar políticas públicas orientadas al fomento de las experiencias autogestionadas y a las producciones locales ${ }^{32}$. Frente a la clausura de milongas, escuelas populares y ateneos en los últimos años, distintos colectivos se organizan ante el grito del \#ElTangoNoSeClausura ${ }^{33}$. La Asociación de Organizadores de Milongas ${ }^{34}$, con el apoyo de otros colectivos, desarrolló una lucha constante contra la clausura arbitraria de milongas por parte del gobierno local, los errores y faltas de la administración y la ausencia de reglamentaciones claras; este proceso culminó en 2016 con la sanción de la Ley de Fomento a las Milongas en la Legislatura porteña (Ley n ${ }^{\circ} 5735$ ). 
Otro ejemplo nos lo ofrece el caso del colectivo Falles Populars i Combatives, que se organiza directamente contra los peligros que consideran puede acarrear una nominación de la Unesco. Este colectivo está formado por lo que se viene denominado movimientos urbanos autónomos (Mompó 2016), caracterizados por una gran heterogeneidad de origen y clase social teniendo en común una misma ideología que pone el acento en la acción y la organización (Dinerstein 2013). Proponen una autonomía frente al capital, el Estado y la hegemonía del desarrollo (Böhm et al. 2010) ${ }^{35}$. Bajo el lema de Patrimoney, se trata de una organización colectiva en forma asamblearia que busca movilizar una nueva perspectiva crítica a la "UNASCO", para concientizar de los riesgos de la nominación como Patrimonio de la Humanidad. Si bien reconocen la dificultad de popularizar la crítica a algo que se entiende generalmente como positivo, se preguntan si no es criticable. Tal y como expresaba un activista "que tenga que venir alguien de no se sabe dónde a decir qué tiene y qué no tiene valor" ${ }^{\prime 36}$. $\mathrm{Su}$ crítica se orienta directamente al discurso oficial del patrimonio, y se estructura ante una experiencia directa de lucha y reivindicación social frente a la ciudad neoliberal, proponiendo recuperar el sentido de la fiesta como vertebrador de sociabilidades y de barrio.

Esta vinculación patrimonio/fiesta/ciudad queda bien reflejada en las canciones oficiales que ha lanzado este colectivo, cada año, para la celebración de las fallas. En el 2016, la canción oficial (Cançó de les \#FPC16) llevó por título: Les falles són populars, combatives des dels solars. En su estribillo se puede escuchar: "las fallas son populares/¡esto es puro disfrute!/Combativas desde los solares/fallas populares y combativas!" 37 . En el 2017, jugando con los términos propios del mundo fallero, la canción se denominó Crema La Ciutat (Cançó de les \#FPC17), literalmente "quema la ciudad", en referencia al acto de la Cremà con el que finalizan las fiestas al prenderse fuego a los monumentos. En esta ocasión, introducían el problema de la turistificación relacionado con la patrimonialización. Su letra ${ }^{38}$ era más agresiva llegándose a decir: "Despierta, dispara, un guiri en casa!", en un juego otra vez metafórico, referido a uno de los aspectos más definitorios de la fiesta, la pólvora. Además, se decía: "estamos hartas de mirar y callar (...) fuego y fiesta, quema la ciudad!". Por último, en el 2018, la Cançó de les \#FPC18, tuvo como lema Les Falles Seran Sempre Nostres, una clara reivindicación a que las fallas no se pueden expropiar, "son siempre nuestras". Además, en este último año, el contenido simbólico era mucho mayor al hacer una adaptación de "A las barricadas", una de las canciones más populares durante la Guerra Civil española vinculada al anarcosindicalismo ${ }^{39}$. Esta articulación entre los procesos patrimoniales y urbanos está muy presente en sus acciones y discursos. En una asamblea de Falles Populars ante la resolución inminente de la Unesco declaraban:

veiem un dels aspectes que ens ha de posar en alerta, més enllà dels riscos de fossilització de la festa que pot comportar la normativització. Incloure les falles $i$ València en l>univers de la Unesco, com ja ha passat en molts altres ocasions, ens porta a posar-la en el mapa internacional de l'excel-lència, del turisme cultural $i$, en el pitjor dels casos, de la ciutat-mercaderia. Si veiem que les falles tenen un valor és, sobretot, el de fer comunitat. El de fer barri. I barri a barri, el de fer ciutat ${ }^{40}$.

Asimismo, como explica uno de sus representantes: "Una casa no puede tener más valor que el que la habita, algo que se puede vincular a las fallas. Uno de los riesgos es que tenga más valor la fiesta que los que la construyen y la viven". En este sentido, la figura de la Patri, tal y como explicaban sus creadores, pretendía ser "un altavoz y canal de comunicación del lema, posición, duda y pensamiento crítico alrededor del proceso patrimonializador que sufre nuestra ciudad y nuestra fiesta".

En los ejemplos a ambos lados del Atlántico, se reivindica un origen popular, independiente, libertario y combativo. En ambos casos se dialoga directamente con el discurso del patrimonio cultural y su rescate. La crítica se estructura en el idioma hegemónico del patrimonio que dio lugar a la globalización del edificio unesquiano: "El tango no se clausura, es nuestro, es Patrimonio de la Humanidad". Sin embargo, ante las versiones edificantes, higienizadas y modélicas se busca recuperar otras memorias luchadoras, narrativas litigantes que abren camino para los discursos reivindicativos del presente. Estos movimientos no se reducen a una perspectiva sobre el pasado, sino que se construyen en gran parte gracias a una proyección hacia una manera de producir nuevos futuros. En Buenos Aires, se reclama, por ejemplo, nuevas leyes de apoyo a las milongas, a los trabajadores del tango y a las experiencias autogestivas, pidiendo la consolidación de las escenas independientes. Mientras tanto, Valencia nos muestra un movimiento social que llama a repensar las fallas desde su carácter más inclusivo y participativo, a tono con las nuevas demandas de democratización de diferentes movilizaciones políticas contemporáneas.

En ningún caso estos movimientos se oponen a las fallas o al tango, sino que buscan esquivar las versiones oficiales que intentan disciplinar y espectacularizar estas expresiones culturales, buscando las otras historias ignoradas. Es desde aquí que se reivindica un carácter popular, por oposición a las apropiaciones elitistas y 
hechas desde el poder; las iniciativas barriales en contra de las versiones globalizadas, deslocalizadas en las que se convierten las expresiones festivas o artísticas de cara a un público global. La espectacularización del tango for export, expresión hecha show que se relame en los voleos de piernas seductoras de medias negras, tiene su paralelismo en las fallas folklorizadas que esconden su espíritu satírico en una demostración monumental e infantilizada. Unesco encarna en muchos casos un último estamento disciplinador de expresiones culturales que ya han sido pasadas por el tamiz de regímenes político de distinto orden y color. Es un último paso que puede vivirse como otra expropiación y que da lugar a reivindicaciones desde su origen más popular, raigambre de protesta que aflora en el corazón de muchos géneros populares. La revuelta, como expresión extrema de la fiesta (Delgado 2001), es un devenir que no ha sido nunca ignorado por el poder, siendo muchos los ejemplos de prohibiciones de fiestas y expresiones artísticas por parte de gobiernos más o menos autoritarios, experiencias por las que han pasado tanto las fallas como el tango.

\section{Conclusión}

Los programas patrimoniales Unesco, lejos de ser asuntos culturales, deben analizarse como proyectos políticos. Los procesos de patrimonialización mundial expresan de forma contundente las relaciones de poder y las políticas nacionales. Los regímenes patrimoniales, en expresión de Bendix (2009), son en realidad regímenes de verdad (Foucault 1992). Pero, mucho más sutiles y enmascarados: la maquinaria patrimonial es la forma de control más edulcorada. La Unesco, empresa por excelencia patrimonial, ha impuesto una versión propia de la globalización a través de la noción de Patrimonio Mundial (Pavone 2008). Su mayor logro ha sido consensuar y presentar como único su sentido de patrimonio (Choay 2007; Labadi 2007; Pavone 2008). Gracias a su legitimación asigna y distribuye, instruye y dosifica, otorga y negocia en un juego de apariencias donde los fines altruistas se imponen ante cualquier otra ficción posible. Pero sus decisiones tienen importantes impactos locales: económicos, sociales, ambientales y culturales. Y su régimen de gobernanza genera tensiones abriendo el conflicto hacia la disputa por los sentidos patrimoniales.

Hoy los futuros patrimoniales más que ensamblados (Harrison 2013) están secuestrados por un mercado cada vez más exigente. La identidad, esa ficción tan líquida como fracturada, permite tonificar las mercancías transnacionales. Los territorios, esos lugares imaginados, luchan por entrar en los circuitos financieros del capitalismo cognitivo. Y las memorias, esos sueños evocados, se convierten en narrativas imposibles de autenticidad. Mientras la Unesco ha dejado de gestionar el patrimonio para gestionar su marca. Sin duda, una empresa mucho más rentable. Y ante esta nueva realidad aparecen voces que reclaman lo propio y su derecho a decidir sobre ello.

Agradecimientos: Agradecemos los comentarios de los evaluadores que nos han permitido introducir mejoras en el texto.

\section{Referencias Citadas}

Anderson, B. 1983. Imagined Communities: Reflections on the Origins and Spread of Capitalism. Verso, Londres.

Anheier, H.K. y Y.R. Isar (eds.) 2008. Cultures and Globalization: The Cultural Economy. Sage, Londres.

Ariño, A. 1992a. La Ciudad Ritual. La Fiesta de las Fallas. Anthropos Editorial, Barcelona.

Ariño, A. 1992b. La fiesta de las fallas. Revista de Antropologia Social 1:29-60.

Ariño, A. (dir.) 1993. Los Escultores del Fuego. Introducción a la Historia del Gremio Artesano de Artistas Falleros de Valencia. Diputació de València, Valencia.

Ashworth, G.J. y B.J. Van der Aa 2006. Strategy and policy for the world heritage convention: goals, practices and future solutions. En Managing World Heritage Sites, editado por A. Leask y A. Fyall, pp.147-158. Elsevier, Burlington.

Askew, M. 2010. The magic list of global status: Unesco, world heritage and the agendas of states. En Heritage and Globalisation, editado por S. Labadi y C. Long, pp. 19-44. Routledge, London.
Azzi, M.1991. Antropología del Tango. Los Protagonistas. Olavarría, Buenos Aires.

Bendix, R.F. 2009. Heritage between economy and politics: An assessment from the perspective of cultural anthropology. En Intangible Heritage, editado por L. Smith y N. Akagawa, pp. 253-269. Routledge, Londres.

Bendix, R.F., A. Eggert y M. Arnika Peselmann (eds.) 2012. Heritage Regimes and the State. Universitätsverlag Göttingen, Göttingen.

Benhamou, F. 2010. L'inscription au patrimoine mondial de l'humanité. Revue Tiers Monde 2:113-130.

Bertoncello, R. y C.A. Troncoso 2014. La ciudad como objeto de deseo turístico: renovación urbana, cultura y turismo en Buenos Aires y Salta (Argentina). Grand Tour 9:4-26.

Boltanski, L. y Ł̀. Chiapello 2002. El nuevo espíritu del capitalismo. Akal, Madrid.

Böhm, S., A.C. Dinerstein y A. Spicer 2010. (Im)possibilities of autonomy: Social movements in and beyond capital, the State and development. Social Movement Studies 9 (1):17-32. 
Boix, R., P. Rausell y R. Abeledo 2017. The Calatrava model: Reflections on resilience and urban plasticity. European Planning Studies 25 (1):29-47.

Bourdieu, P.1988. La Distinción. Criterio y Bases Sociales del Gusto. Taurus, Madrid.

Bourdieu, P.1991. El Sentido Práctico. Taurus, Madrid.

Bourdieu, P. 1994. Raisons Pratiques: Sur la Théorie de l'action. Seuil, París.

Brumann, C. 2012. Multilateral Ethnography: Entering the World Heritage Arena. Max Planck Institute for Social Anthropology, Working Paper 136.

Cal, R. 2007. La propaganda del turismo en España. Primeras organizaciones. Historia y Comunicación Social 2:125-133.

Carrero, V., R.M. Soriano y A. Trinidad 2006. Teoría Fundamentada Grounded Theory. La Construcción de la Teoría a través del Análisis Interpretacional. CIS, Madrid.

Cecconi, S. 2009. Territorios del tango en Buenos Aires: aportes para una historia de sus formas de inscripción. Iberoamericana 9 (33):49-68.

Choay, F. 1996. L'allégorie du patrimoine. Seuil, París.

Collins, J. 2008. But what if I should need to defecate in your neighbourhood, Madame: Empire, redemption, and the "Tradition of the Oppressed" in a Brazilian World Heritage Site. Cultural Anthropology 23 (2):279-328.

Comaroff, J.L. y J. Comaroff 2009. Etnicidad S.A. Katz, Madrid.

Connolly, M. 2013. The 'Liverpool model(s)': Cultural planning, Liverpool and capital of culture 2008. International Journal of Cultural Policy 19 (2):1-20.

Cruces, F. 1998. Problemas en torno a la restitución del patrimonio. Una visión desde la antropología. Política y Sociedad 27:77-87.

Cucó. J. 2008. Sociabilidades urbanas. Ankulegi 12 (2008):65-82.

Cucó, J. (ed.) 2013a. La Ciudad Pervertida: Una Mirada sobre la Valencia Global. Anthropos Editorial, Barcelona.

Cucó, J. (ed.) 2013b. Metamorfosis Urbanas. Ciudades Españolas en la Dinámica Global. Icaria, Barcelona.

Cuenya, B. 2011. Grandes proyectos y sus impactos en la centralidad urbana. Carajillo de la Ciudad 13 (25):185-212.

Cuenya, B. y M. Corral 2011. Empresarialismo, economía del suelo y grandes proyectos urbanos: el modelo de Puerto Madero en Buenos Aires. EURE 37 (111): 25-45.

Davallon, J. 2010. The game of heritagization. En Constructing Cultural and Natural Heritage. Parks, Museums and Rural Heritage, editado por X. Roigé y J. Frigolé, pp. 27-38. ICRPC, Girona.

De Cesari, C. 2010. Creative heritage: Palestinian heritage NGOs and defiant arts of government. American Anthropologist 112 (4):625-637.

Delgado, M. 2001. Carrer, festa i revolta. Els usos simbòlics de 1'espai públic a Barcelona (1951-2000). Revista d'etnologia de Catalunya 18:143-145.

Díaz Orueta, F. 2010. Regímenes urbanos y movimiento ciudadano en Valencia. Cuaderno Urbano 9 (9):275-294.

Díaz Orueta, F., M.L. Lourés, C. Rodríguez y V. Devalle 2003. Ciudad, territorio y exclusión social. Las políticas de recualificación urbana en la ciudad de Buenos Aires. Reis 159-185.

Dinardi, C. 2017. Cities for sale: Contesting city branding and cultural policies in Buenos Aires. Urban Studies 54 (1):85-101.
Dinerstein, A.C. (ed.) 2013. Movimientos Sociales y Autonomía Colectiva: La Política de la Esperanza en América Latina. Capital Intelectual, Buenos Aires.

Escobar, A. 2010. Una Minga para el Postdesarrollo: Lugar, Medio Ambiente y Movimientos Sociales en las Transformaciones Globales. Universidad Nacional Mayor de San Marcos, Lima.

Foucault, M. 1992. Microfísica del Poder. Endymión, Madrid.

Frey, B.S., P. Pamini y L. Steiner 2013. Explaining the world heritage list: An empirical study. International Review of Economics 60 (1):1-19.

Frigolé, J. 2014. Retóricas de la autenticidad en el capitalismo avanzado. Endoxa 33:37-60.

García, B. 2004. Cultural policy and urban regeneration in western European cities: Lessons from experience, prospects for the future. Local Economy 19 (4):312-326.

García Canclini, N. 1993. Los usos sociales del patrimonio cultural. En El Patrimonio Cultural de México, compilado por E. Florescano, pp. 41-62. FCE, México DF.

Glass, R. 1964. Aspects of Change. MacKibbon and Kee, Centre for Urban Studies, Londres.

Gold, J. y M Gold 2008. Olympic cities: Regeneration, city rebranding and changing urban agendas. Geography Compass 2 (1):300-318.

Gómez, M., A. Almirón y M. González 2011. La cultura como recurso turístico de las ciudades: El caso de la patrimonialización del tango en Buenos Aires, Argentina. Estudios y Perspectivas en Turismo 20 (5):1027-1046.

Harrison, R. 2013. Heritage: Critical Approaches. Routledge, Londres.

Harvey, D. 1998. La Condición de la Posmodernidad. Investigación sobre los Orígenes del Cambio Cultural. Amorrurtu, Buenos Aires.

Harvey, D. 2001. Spaces of Capital: Towards a Critical Geography. Edinburgh University Press, Edinburgh.

Harvey, D. 2013. Ciudades Rebeldes: del Derecho de la Ciudad a la Revolución Urbana. Akal, Madrid.

Heinich, N. 2009. La Fabrique du Patrimoine. De la Cathédrale à la Petite Cuillère. Maison des Sciences de 1'Homme, París.

Hernàndez, G.M. 1995. El reinvent d'una tradició. La festa de les Falles sota el franquisme. El Contemporani: Revista d'Història 6:54-62.

Hernàndez, G.M. 1996. Falles i Franquisme a València. Afers, Catarroja.

Hernàndez, G.M. 2006. El triunfo de la tradición flexible. La celebración de la identidad fallera a través de la Ofrenda de Flores a la Virgen de los Desamparados de Valencia. Zainak. Cuadernos de Antropología-Etnografía 28:125-146.

Hernàndez, G.M. 2008. Los estudios falleros. El desarrollo de la investigación social sobre las Fallas de Valencia. ANDULI, Revista Andaluza de Ciencias Sociales 6:93-114.

Hernàndez, G.M. 2011. Focs de Falla. Articles per al Combat Festiu. Obrapropia, Valencia.

Herzfeld, M. 1991. A Place in History: Social and Monumental Time in a Cretan Town. Princeton University Press, Princeton.

Labadi, S. 2007. Representations of the nation and cultural diversity in discourses on World Heritage. Journal of Social Archaeology 7 (2):147-170.

Lowenthal, D. 1998. The Heritage Crusade and the Spoils of History. Cambridge University Press, Cambridge. 
MacCannell, D. 1999. The Tourist: A New Theory of the Leisure Class. University of California Press, California.

MacDonald, S. 2013. Memorylands. Heritage and Identity in Europe Today. Routledge, Londres.

Mármol, C. Del 2012. Pasados Locales, Políticas Globales. Germania-AVA, Valencia.

Matamoro, B. 1982. La Ciudad del Tango Histórico y Sociedad. Editorial Galerna, Buenos Aires.

Meskell, L. 2013. Unesco's world heritage convention at 40 Challenging the economic and political order of international heritage conservation. Current Anthropology 54 (4):483-494.

Meskell, L. 2014. States of conservation: Protection, politics, and pacting within Unesco's world heritage committee. Anthropological Quarterly 87 (1):217-243.

Meskell, L., C. Liuzza, E. Bertacchini y D. Saccone 2015. Multilateralism and Unesco World Heritage: decision-making, States Parties and political processes. International Journal of Heritage Studies 21 (5):423-440.

Meskell, L. y C. Brumann 2015. Unesco and new world orders. En Global Heritage: A Reader, editado por L. Meskell, pp 22 42. Wiley Blackwell, Chichester.

Mompó, E. 2016. Contra-narrativas y prácticas de resistencia en un conflicto urbano. Una respuesta autónoma desde El Cabanyal (Valencia). Actas del Congreso Internacional Contested Cities (Serie IV-5B). Artículo n ${ }^{\circ}$ 5-543. En http://contested-cities.net/ working-papers/autor/eva-mompo/

Morel, H. 2009. El giro patrimonial del tango: políticas oficiales, turismo y campeonatos de baile en la ciudad de Buenos Aires. Cuadernos de Antropología Social 30:155-172.

Morel, H. 2013. Buenos Aires la meca del tango: procesos de activación, megaeventos culturales, turismo y dilemas en el patrimonio local. PUBLICAR-En Antropología y Ciencias Sociales 15:55-74.

Morel, H. 2016. Milongas barriales en la ciudad de Buenos Aires: sentidos de lugar, sociabilidad y tradiciones. Etnográfica. Revista do Centro em Rede de Investigação em Antropologia 20 (3):517538.

Obiol, E. 2009. Geografía del Turismo en Valencia. Notas históricas. Los fundamentos del desarrollo turístico. En La Ciudad de Valencia, Historia, Geografía y Arte, editado por J. Hermosilla, pp. 91 -103. PUV, Valencia.

Pavone, V. 2008. From the Labyrinth of the World to the Paradise of the Heart: Science and Humanism in Unesco's Approach to Globalization. Lexington, New York.

Pelinski, R. 2000. El tango nómade. En El Tango Nómade. Ensayos sobre la Diáspora del Tango, compilado por R. Pelinski, pp. 27-70. Corregido, Buenos Aires.

Poulot, D. 2006. Une Histoire du Patrimoine en Occident, XVIII-XIX Siècle. Du Monuments aux Valeurs. Presses Universitaires de France, París.
Prats, Ll. 1997. Antropología y Patrimonio. Ariel, Barcelona.

Rausell, P. 2006. Consideraciones globales hacia el tránsito de Valencia como una ciudad global. Ciudades 71:49-57.

Rausell, P. 2010. Valencia desde la huerta al ocio. En Valencia, 19572007. De la Riada a la Copa del América, editado por J. Sorribes, pp. 79-100. Publicacions de la Universitat de València, Valencia.

Rius, J., G.M. Hernàndez y F. Torres 2016. Urban development and cultural policy "White Elephants": Barcelona and Valencia. European Planning Studies 24(1):61-75.

Rodríguez, A. y L. Vicario 2005. Innovación, competitividad y regeneración urbana: los espacios retóricos de la 'ciudad creativa' en el nuevo Bilbao. Ekonomiaz 58 (1):262-295.

Roigé, X. y J. Frigolé (eds.) 2010. Constructing Cultural and Natural Heritage. Parks, Museums and Rural Heritage. ICRPC, Girona.

Sánchez-Carretero, C. 2012. Hacia una antropología del conflicto aplicada al patrimonio. En Geopolíticas Patrimoniales: De Culturas, Naturalezas e Inmaterialidades. Una Mirada Etnográfica, editado por B. Santamarina Campos, pp. 195-210. Germania, Valencia.

Santamarina, B. y O. Beltran 2016. Heritage and knowledge. Apparatus, logic and strategies in the formation of heritage. Anthropological Forum 26 (4):397-414.

Santamarina, B. y C. Del Mármol 2017. Ciudades creativas y pueblos con encanto: los nuevos procesos patrimoniales del siglo XXI Revista de Dialectología y Tradiciones Populares 72 (2):359-377.

Santamarina, B. y A. Moncusí 2015. El mercado de la autenticidad: las nuevas ficciones patrimoniales. Revista de Occidente 440-441: 93-112

Schmitt, T.M. 2008. The Unesco concept of safeguarding intangible cultural heritage: Its background and Marrakchi roots. International Journal of Heritage Studies 14:95-111.

Scott, A. J. 2008. Social Economy of the Metropolis: CognitiveCultural Capitalism and the Global Resurgence of Cities. Oxford University Press, Oxford.

Scott, A.J. 2014. Beyond the creative city: Cognitive-cultural capitalism and the new urbanism. Regional Studies 48 (4):565-578.

Smith, L. 2006. Uses of Heritage. Routledge, Nueva York.

Swyngedouw, E., F. Moulaert y A. Rodriguez 2002. Neoliberal urbanization in Europe: large-scale urban development projects and the new urban policy. Antipode 34 (3):542-577.

Throsby, D. 2001. Economics and Culture. Cambridge University Press, Cambridge.

UNWTO. 2017. Annual Report 2016. http://cf.cdn.unwto.org/ sites/all/files/pdf/annual_report_2016_web_0.pdf

Varela, G. 2005. Mal de Tango: Historia y Genealogía Moral de la Música Ciudadana. Paidós, Buenos Aires.

Vila, P. 2000. El tango y las identidades étnicas en Argentina. En El Tango Nómade. Ensayos sobre la Diáspora del Tango, compilado por R. Pelinski, pp. 71-80. Corregido, Buenos Aires. 


\section{Notas}

1 "El tango fue declarado patrimonio de la humanidad", Página 12, 30 de setiembre de 2009. https://www.pagina12.com.ar/ diario/ultimas/20-132657-2009-09-30.html

${ }^{2}$ En las Torres se inicia cada año la fiesta con la crida. La crida es el término valenciano utilizado por el mundo fallero para este acto y se puede traducir por llamamiento.

3 "Las Fallas ya son Patrimonio de la Humanidad de la UNESCO", Levante, 01/12/2016. http://www.levante-emv.com/fallas/2016/11/30/ fallas-patrimonio-humanidad-Unesco/1498820.html

${ }^{4}$ El Café Vinilo es un espacio cultural situado en el barrio de Palermo (Buenos Aires) abierto en 2009. Desde su abertura se ha caracterizado por ofrecer conciertos de distintos géneros alternativos.

${ }_{5}^{5}$ Disponible en: http://tangoyculturapopular.blogspot.com/2009/10/ tango-y-cultura-popular-n-108.html

${ }^{6}$ La respuesta de los medios más conservadores no se hizo esperar. El periódico ABC, de tirada nacional, criticaba en una noticia "la campaña contra las fallas y la Unesco" tras iniciarse el proyecto de la Patri (ABC, 27/01/2016). https:// www.abc.es/espana/comunidad-valenciana/abci-empresamusical-campana-fallas-201601271030_noticia.html

7 Todavía se puede visitar la campaña en https://www.verkami. com/projects/13938-falles-populars-i-combatives-2016

${ }^{8}$ Más información sobre el proyecto: https://crearqcio.com/ portfolio/la-patrimonialitzadora/

${ }^{9}$ Este colectivo se define en su página web de la siguiente manera: "Las Fallas Populares y Combativas somos una propuesta de fiesta abierta y autogestionada nacida el 2002 en el distrito de Ciutat Vella fruto del trabajo conjunto de varios colectivos de la ciudad de Valencia" (https://fallespopulars.org/).

${ }^{10}$ Recordemos que las convenciones son tratados multilaterales, con carácter vinculante para los estados firmantes tanto en periodo de paz como de guerra.

${ }^{11}$ Para una revisión sobre el conflicto y el patrimonio ver SánchezCarretero (2012).

${ }^{12}$ Para un acercamiento al tango ver, entre otros: Azzi (1991), Matamoro (1982), Pelinski (2000), Varela (2005).

${ }^{13}$ Se calcula que, aproximadamente, se plantan en Valencia 700 fallas (entre las fallas infantiles y las mayores).

${ }^{14}$ Para una aproximación a las fallas ver, entre otros, a Ariño (1992a, 1992b, 1993) y Hernàndez (1995,1996, 2006, 2008, 2011).

${ }^{15}$ En este caso, trabajamos solo lo sucedido en Argentina porque fue la impulsora del primer intento de inscribir el tango en el programa de la Unesco (2001).

${ }^{16}$ Dejamos de lado, expresamente, el debate sobre su internacionalización a partir de su éxito en París en los años veinte. Para una discusión sobre los procesos y narrativas en torno a los itinerarios del tango se puede acudir a Morel (2012). ${ }^{17}$ Disponible http://servicios.infoleg.gob.ar/infolegInternet/verNorma. do?id=187292
${ }^{18}$ Disponible http://www2.cedom.gob.ar/es/legislacion/normas/ leyes/ley $130 . h t m l$

${ }^{19}$ El Estado español impulsó sus primeras políticas patrimoniales y turísticas, ambas inseparables, a comienzos del siglo XX (Cal 1997).

${ }^{20}$ Disponible http://www.boe.es/datos/pdfs/BOE/1946/075/ A02058-02061.pdf

${ }^{21}$ Orden del 30 de septiembre de 1964, Boletín Oficial del Estado ${ }^{\circ} 251$

${ }^{22}$ Disponible https://www.boe.es/boe/dias/1965/02/10/pdfs/ A02168-02168.pdf

${ }^{23}$ Disponible https://www.boe.es/boe/dias/1980/02/16/pdfs/ A03783-03784.pdf

${ }^{24}$ Disponible http://www.dogv.gva.es/datos/2012/03/12/pdf/2012_2536. pdf

${ }^{25}$ En este sentido, la Unesco refleja las estructuras de poder de otros organismos mundiales (Frey et al. 2013; Meskell et al. 2015).

${ }^{26}$ Desde que Glass acuñara este término en 1964 para los procesos de renovación residencial éste ha sido pródigamente utilizado sufriendo ampliaciones y nuevas acepciones. Sin discutir lo acertado o no de su uso, la autora lo aplica para referirse a la elitización del tango.

${ }^{27}$ Información disponible en http://fallas.com/index.php/es/

${ }^{28}$ Obviamente, las propias particularidades socioeconómicas de cada contexto explican el alcance y el impacto de este proceso. Apuntamos sólo tendencias globales con resultados desiguales en cada realidad particular.

29 "El tango ya no es nuestro", La Nota Publicación del Sindicato Argentino de Músicos y de la Obra Social de Músicos, diciembre de 2009. https://studylib.es/doc/8073494/diciembre-2009

${ }^{30}$ Ver https://www.quintetonegrolaboca.com.ar/presentacion.html

${ }^{31}$ Entrevista con un periodista especializado en tango, 2014.

${ }^{32} \mathrm{http}$ ///orquestodromo.com.ar/festival/index.php/nuestrosvalores/

${ }^{33}$ Entrevista "Escuela Popular de Tango de la Boca" TV Pública, programa Visión 7. 2010. Disponible en https:// www.youtube.com/watch? $\mathrm{v}=\mathrm{xMbocpthXUE}$.

${ }^{34} \mathrm{http}$ ://milongas.org.ar/

${ }^{35}$ Este colectivo no es el único que ha efectuado una crítica sobre las políticas Unesco. Desde la academia e incluso desde dentro de la institución, como ya hemos señalado, cada vez hay más disenso.

${ }^{36}$ Entrevista Carlos Soler en http://valenciaplaza.com/las-fallascomo-patrimonio-de-la-humanidad-las-voces-en-contra

${ }^{37} \mathrm{Se}$ han traducido por las autoras todas las letras al castellano para ayudar al lector. Videoclip disponible en https://www. youtube.com/watch?v=JGIgKRcnii0

${ }^{38}$ Videoclip disponible en https://www.youtube.com/ watch? $\mathrm{v}=\mathrm{ODbCbundIjU}$

${ }^{39} \mathrm{https}: / / \mathrm{www} . y o u t u b e . c o m / w a t c h ? \mathrm{v}=\mathrm{s} 1 \mathrm{GTKTn} 2 \mathrm{Lrc}$

${ }^{40}$ Valencia Plaza, 01/12/2016. Disponible en https://valenciaplaza. com/la-festa-que-volem 
